\title{
Información, motivación y habilidades conductuales asociadas al uso del condón en la última relación sexual en hombres privados de la libertad*
}

\author{
Ricardo Sánchez Medina \\ David Javier Enríquez Negrete \\ Consuelo Rubí Rosales Piña \\ Universidad Nacional Autónoma de México, \\ Ciudad de México
}

\begin{abstract}
Recibido: 31 de enero del 2019 / Aceptado: 4 de marzo del 2019 doi: 10.26439/persona2019.n022(1).4082
\end{abstract}

El objetivo del estudio fue analizar en hombres privados de la libertad, pertenecientes a tres estratos de edad, las diferencias sobre el uso del condón con respecto al nivel de información sobre el VIH, la motivación y las habilidades conductuales en su utilización. Se evaluó a un total de 1104 varones de comunidades de diagnóstico y de un centro de reinserción social de Ciudad de México. Fueron divididos en tres grupos, empleando como criterio la distribución asignada por los cuartiles de la edad, así como la comparación entre quienes usaron o no usaron condón en su última relación sexual. Se realizó un análisis factorial 3x2, en el que se encontraron diferencias en el grado de información y en las habilidades conductuales, en relación con los grupos de edad y la utilización del condón; sin embargo, la motivación no mostró diferencias significativas. Los resultados se discuten en términos de la importancia de diseñar programas de intervención en hombres privados de la libertad, en los que se considere cómo la experiencia se relaciona con el uso del condón en relaciones sexuales.

hombres / condón / entorno carcelario / grupo etario / prevención VIH

* Investigación realizada gracias al Programa UNAM-DGAPA-PAPIIT IA304317.

Correo electrónico: risame81@gmail.com 


\section{Information, motivation and behavioral skills associated with condom use in the last sexual intercourse in males deprived of freedom}

This study aimed to analyze the differences in condom use with respect to the level of information about HIV, and the motivation and behavioral skills to use condoms in males deprived of freedom from three age strata. A total of 1,104 males belonging to long-term pretrial detention facilities for adolescents (comunidades de diagnóstico) and a social rehabilitation center in Mexico City were evaluated. They were divided into three groups using as criteria the distribution assigned by age quartiles, as well as the comparison between those who used and those who did not use a condom in their last sexual intercourse. A $3 \times 2$ factorial analysis was carried out and differences were found regarding the level of information and behavioral skills in relation to the age groups and condom use; however, there were no significant differences in the motivation. The results are discussed in terms of the importance of designing intervention programs for males deprived of freedom considering how the experience is related to the use of condoms in sexual intercourse. 


\section{INTRODUCCIÓN}

De acuerdo con la Organización Panamericana de la Salud (2018), los problemas relacionados con la salud sexual en jóvenes son variados y complejos. Uno de ellos tiene que ver con la adquisición de infecciones de transmisión sexual (ITS), entre las que destaca el VIH/SIDA, debido a las conductas sexuales de riesgo y a las barreras culturales que pueden limitar un comportamiento preventivo.

En el ámbito internacional, el número de personas infectadas por VIH en el 2017 fue de 36,9 millones, de las cuales 21,7 millones tuvieron acceso a un tratamiento. Durante ese mismo año 1,8 millones de personas contrajeron la infección y de ellas fue mayor la proporción de hombres que de mujeres (Programa Conjunto de las Naciones Unidas sobre el VIH/SIDA, 2018).

Autores como Cruz, Ramos y González (2011), y Canche, Canul, Suárez, De Anda, y González (2011) mencionan que estas infecciones se asocian a diversos factores tanto biológicos como conductuales y contextuales. Por ejemplo, señalan que las mujeres son más susceptibles a la infección, al no usar preservativo en las relaciones sexuales y estar expuestas a contextos de riesgo.

Si bien las mujeres son más susceptibles a la infección, se ha encontrado que en su mayoría son contagiadas por sus parejas varones (Amuchástegui, 2017). En el caso particular de los hombres, ellos presentan más conductas de riesgo sexual, en comparación con las mujeres (Folch et al., 2015). Lo anterior se complica cuando las relaciones sexuales se dan en contextos de riesgo como el carcelario. Ahí se registra mayor prevalencia de infección por VIH que en la población en general (Bórquez et al., 2017) y una mayor presencia de conductas de riesgo (Sánchez, Rosales y Enríquez, 2018), como relaciones sexuales sin protección y poca accesibilidad a condones (Adoga et al., 2009; Gough y Edwards, 2009).

Una explicación de por qué las personas adoptan conductas que ponen en riesgo su salud se encuentra en el Modelo de Información-Motivación-Habilidades conductuales (IMB, por sus siglas en inglés) propuesto por Fisher y Fisher (1992). En este se considera que deben analizarse tres factores para que una persona use condón en sus relaciones sexuales: información, referida al conocimiento sobre prevención de VIH y formas en las que se contagia; motivación, entendida en este modelo como creencias, actitud e intención que tiene acerca del uso del condón para la prevención del VIH; y habilidades conductuales, es decir, lo que hace para protegerse, como hablar con la pareja y negociar el uso del condón en relaciones sexuales.

Desde el modelo IMB, se ha encontrado que es más probable que se protejan y usen el preservativo los hombres con información de cómo protegerse, motivados para usar condón y con las habilidades para hacerlo (Bermúdez, Herencia Leva y Uribe, 2009; Cunill, Gras, Planes y Serda, 2012; Espada, Morales, Guillén, Ballester y Orgilés, 2016; Liu, et al., 2014; Mazo, Domínguez y Cardona, 2014; Uribe, Aguilar, Zacarías y Aguilar, 2015).

Partiendo de que la prevalencia de infección por VIH es más alta en la población 
privada de la libertad que en la población en general y que los hombres presentan más conductas de riesgo sexual en comparación con las mujeres, es necesario identificar qué lleva a estas personas a usar condón en sus relaciones sexuales, con el fin de generar intervenciones para promover la salud sexual en estos contextos de mayor vulnerabilidad.

La literatura reporta que entre más temprano los jóvenes inicien su vida sexual, más probable es que asuman conductas de riesgo sexual (Cueto y Leon, 2016) y delictivas (López, Garrido, López, López y Galvis, 2016); de esta manera la experiencia adquirida a lo largo de la vida puede influir en la presencia o ausencia de conductas de riesgo. Con base en lo anterior, el objetivo de esta investigación es analizar si la información, la motivación y las habilidades conductuales se asocian con el uso o no uso del condón en la última relación sexual, para lo cual se compara a hombres en diferentes estratos de edad con el fin de identificar qué los lleva a usar preservativo en sus relaciones sexuales.

\section{MÉtodo}

\section{Participantes}

Se trabajó con 1104 hombres privados de la libertad, pertenecientes a comunidades de diagnóstico y tratamiento para adolescentes y al Centro Varonil de Reinserción Social Santa Martha Acatitla: 417 de las comunidades y 687 del centro varonil. A través de un muestreo intencionado (Kerlinger y Lee, 2002) se invitó a los usuarios a participar. Como criterio de inclusión se trabajó con hombres delincuentes primarios, con índice criminal bajo y medio, con cualquier delito de fuero común y portación de arma de fuego; como criterio de exclusión, población sentenciada y ejecutoriada, así como personas con trastornos mentales. La muestra se dividió en tres grupos de edades diferentes, utilizando como criterio la distribución asignada por los cuartiles de la edad. El primer grupo quedó conformado por 347 adolescentes con una edad promedio de 16,17 años ( $\mathrm{SD}=1,02)$; el segundo grupo, por 430 jóvenes con una edad promedio de 22,90 años ( $\mathrm{SD}=2,56)$; y el tercer grupo, por 428 adultos de 32,30 años en promedio $(\mathrm{SD}=8,14)$. En la tabla 1 se muestran las características sociodemográficas de los grupos.

\section{Instrumentos}

- Prueba de Conocimientos sobre VIH/ SIDA-ITS (Robles y Díaz-Loving, 2011). Son 12 reactivos relacionados con conocimientos sobre VIH/SIDA y 6 reactivos referentes al uso correcto del condón. Los reactivos tienen un formato de respuesta de cierto, falso y no sé. La prueba tiene un índice de dificultad de 0,52.

- Prueba de Conocimientos sobre Uso de Condón (Robles y Díaz-Loving, 2011). Contiene 6 reactivos relacionados con el uso correcto del condón. Los reactivos tienen un formato de respuesta de cierto, falso y no sé. La prueba tiene un índice de dificultad de 0,60.

- Escala de Creencias hacia el Uso del Condón (Robles y Díaz-Loving, 2011). Comprende 8 reactivos que evalúan creencias favorables y 4 que evalúan creencias negativas sobre el uso del 
Tabla 1

Comparación entre grupos sobre variables sociodemográficas

\begin{tabular}{|c|c|c|c|c|c|c|c|}
\hline \multirow[t]{2}{*}{ Característica } & \multicolumn{2}{|c|}{$\begin{array}{c}\text { Grupo } \\
\text { de adolescentes } \\
(n=341)\end{array}$} & \multicolumn{2}{|c|}{$\begin{array}{c}\text { Grupo } \\
\text { de jóvenes } \\
(n=417)\end{array}$} & \multicolumn{2}{|c|}{$\begin{array}{c}\text { Grupo } \\
\text { de adultos } \\
(n=346)\end{array}$} & \multirow[t]{2}{*}{$\begin{array}{l}\text { Prueba } \\
\text { estadística }\end{array}$} \\
\hline & $\mathrm{N}$ & $\%$ & $\mathrm{~N}$ & $\%$ & $\mathrm{n}$ & $\%$ & \\
\hline Estado civil & & & & & & & $x^{2}=91.364 p<.001$ \\
\hline Soltero & 294 & 86,5 & 292 & 70,2 & 196 & 57,8 & \\
\hline Casado & 1 & 0,3 & 30 & 7,2 & 51 & 15,0 & \\
\hline Unión libre & 45 & 13,2 & 88 & 21,2 & 81 & 23,9 & \\
\hline Otro & 0 & 0,0 & 6 & 1,4 & 11 & 3,2 & \\
\hline Nivel de estudios & & & & & & & $x^{2}=52.864 p<.001$ \\
\hline Ninguno & 15 & 4,4 & 14 & 3,4 & 10 & 3,0 & \\
\hline Primaria & 120 & 35,4 & 88 & 21,5 & 63 & 18,9 & \\
\hline Secundaria & 179 & 52,8 & 253 & 61,9 & 204 & 61,3 & \\
\hline Preparatoria & 24 & 7,1 & 53 & 13,0 & 44 & 13,2 & \\
\hline Universidad & 1 & 0,3 & 1 & 0,2 & 12 & 3,6 & \\
\hline Tiene hijos & & & & & & & $x^{2}=201.677 p<.001$ \\
\hline Sí & 44 & 12,9 & 171 & 42,1 & 225 & 66,4 & \\
\hline No & 296 & 87,1 & 235 & 57,9 & 114 & 33,6 & \\
\hline Ingresos familiares & & & & & & & $x^{2}=44.2 p<.001$ \\
\hline Menos de $\$ 2000$ & 67 & 19,8 & 91 & 22,4 & 101 & 30,0 & \\
\hline De 2001 a 3999 & 60 & 17,8 & 75 & 18,4 & 56 & 17,1 & \\
\hline De 4000 a 5999 & 34 & 10,1 & 51 & 12,5 & 53 & 16,2 & \\
\hline De 6000 a 9999 & 19 & 5,6 & 31 & 7,6 & 25 & 7,6 & \\
\hline Más de 10000 & 12 & 3,6 & 23 & 5,7 & 20 & 6,1 & \\
\hline No sabe & 146 & 43,2 & 134 & 32,9 & 72,0 & 22,0 & \\
\hline Delito cometido & & & & & & & $X^{2}=52.099 p<.001$ \\
\hline Robo & 270 & 79,4 & 313 & 76,7 & 281 & 84,1 & \\
\hline Extorsión & 0 & 0,0 & 11 & 2,7 & 4 & 1,2 & \\
\hline Lesiones & 11 & 3,2 & 7 & 1,7 & 1 & 0,3 & \\
\hline Violación & 5 & 1,5 & 9 & 2,2 & 12 & 3,6 & \\
\hline Homicidio & 22 & 6,5 & 31 & 7,6 & 10 & 3,0 & \\
\hline $\begin{array}{l}\text { Privación ilegal } \\
\text { de la libertad }\end{array}$ & 20 & 5,9 & 13 & 3,2 & 1 & 0,3 & \\
\hline $\begin{array}{l}\text { Delincuencia } \\
\text { organizada }\end{array}$ & 3 & 0,9 & 3 & 0,7 & 3 & 0,9 & \\
\hline Otras causas & 9 & 2,6 & 21 & 5,1 & 22 & 6,6 & \\
\hline
\end{tabular}


condón con pareja regular, en una escala tipo Likert de 1 (totalmente en desacuerdo) a 5 (totalmente de acuerdo). La consistencia interna es de $\alpha=.84$.

- Escala de Actitudes hacia el Uso del Condón (Robles y Díaz-Loving, 2011). Comprende 5 reactivos con opciones de respuesta definidas en una escala de diferencial semántico de 1 a 7 (por ejemplo, placentero - no placentero). La escala se aplica para evaluar actitudes con pareja regular y con pareja ocasional. La consistencia interna es de $\alpha=.87$.

- Intención para Usar Condón en Relaciones Sexuales (Robles y Díaz-Loving, 2011). Se evalúa a través del reactivo “¿Qué tan probable es que la próxima vez que tengas relaciones sexuales con tu pareja estable uses condón?" en escala de 7 puntos $(1=$ nada probable; $7=$ totalmente probable).

- Autoeficacia para Usar Condón en Relaciones Sexuales (Robles y Díaz-Loving, 2011). Es 1 reactivo en una escala de 1 a 7 que evalúa “¿Qué tan seguro estás de que puedas usar un condón cada vez que tienes sexo vaginal con tu pareja estable?", en el que 1 es muy seguro de no poder usarlo y 7 es muy seguro de poder usarlo.

- Escala de Comunicación sobre Temas Sexuales con la Pareja (Robles y DíazLoving, 2011). Contiene 10 reactivos que miden la frecuencia con que se comunican con su pareja sobre temas sexuales, con valores de 1 (nunca) a 5 (siempre). Su consistencia interna es de $\alpha=.94$.
- Inventario de Estilos de Negociación sobre el Uso del Condón (Robles y Díaz-Loving, 2011). Comprende 6 reactivos que miden el estilo de colaboración y equidad, y 3 que miden el estilo de contender, referidos a negociar el condón con su pareja, con valores de 1 (totalmente en desacuerdo) a 5 (totalmente de acuerdo). La consistencia interna del estilo de colaboración-equidad es $\alpha=0,88$, y del estilo contender es $\alpha=.59$.

\section{Procedimiento}

Para la recolección de los datos, se hizo una invitación a los hombres que integran cada centro; en el caso de los menores de edad, se solicitó consentimiento informado de los padres. A los que aceptaron participar se les entregó un consentimiento informado, en el cual se les explicó la confidencialidad de la información, así como la participación voluntaria. Posteriormente, se les dio el instrumento y un lápiz. El tiempo que tardaron en contestarlo fue de aproximadamente 1 hora. En ambas instituciones se realizaron talleres para promover la salud sexual entre la población.

Para el análisis de resultados se utilizó un diseño factorial $3 \times 2$; de esta forma, se formaron 6 grupos de comparación en términos de la edad y del uso del condón en la última relación sexual (ver tabla 2). Se utilizó la prueba estadística Anova factorial, en la que se reportaron las $F$ de los factores principales (grupos de edad y uso de condón en última relación sexual) sobre las variables del modelo IMB, así como la eta cuadrada $\left(\eta^{2}\right)$. 
Tabla 2

Grupos formados a partir del diseño factorial 3x2 para la investigación

\begin{tabular}{lcccc}
\hline \multirow{2}{*}{ Adolescentes } & \multicolumn{3}{c}{ Grupos de edad } \\
\cline { 2 - 4 } & & Jóvenes & Adultos & \\
\cline { 2 - 4 } $\begin{array}{l}\text { Uso de condón } \\
\text { en última relación } \\
\text { sexual }\end{array}$ & Sí & 1 & 2 & 3 \\
\hline
\end{tabular}

\section{Resultados}

En la tabla 3 se presentan las medias obtenidas sobre el nivel de conocimientos con respecto al uso de condón en la última relación sexual y los grupos de edad, así como las puntuaciones obtenidas con Anova factorial y eta cuadrada. Se observaron diferencias significativas en términos de los grupos de edad, pero no sobre el uso del preservativo; sin embargo, únicamente se encontró un efecto de interacción entre la utilización del condón y los grupos de edad sobre los conocimientos de VIH/SIDA. Se aplicó una prueba post hoc para identificar entre qué grupos de edad se daban las diferencias. Al aplicar la prueba de Bonferroni, se encontraron diferencias significativas entre el grupo de adolescentes y jóvenes ( $\mathrm{I}-\mathrm{J}=0,53, \mathrm{p}<.007$ ), así como adolescentes y adultos $(\mathrm{I}-\mathrm{J}=.63, \mathrm{p}<.001)$; pero no hubo diferencias entre jóvenes y adultos en los conocimientos sobre VIH/SIDA. Estos resultados indican que el nivel de conocimientos sobre VIH/SIDA varía en términos de los que usaron o no usaron condón en su última relación sexual y la edad. Acerca de los conocimientos sobre uso del condón, se aplicó una prueba post hoc para identificar entre qué grupos había diferencias. Así, al aplicar la prueba de Bonferroni, únicamente se encontraron diferencias significativas entre el grupo de adolescentes y adultos $(\mathrm{I}-\mathrm{J}=.33, \mathrm{p}<.007)$. Esto indica que es mayor el conocimiento sobre este tema en adultos, pero no hay una relación de interacción entre edad y uso de condón sobre lo que saben del preservativo.

En cuanto al componente de motivación, en la tabla 4 se muestran las medias de los grupos respecto de quienes usaron condón en su última relación sexual y los grupos de edad, así como la Anova factorial y la eta cuadrada. En términos generales, no se encontraron diferencias significativas; únicamente, las hubo sobre creencias negativas y actitudes hacia el uso de condón entre quienes lo usaron o no lo usaron en sus relaciones sexuales. Esto quiere decir que los que utilizaron el preservativo tienen menos creencias negativas de su empleo, así como actitudes más favorables para usarlo. En ninguna de las variables evaluadas se encontró un efecto de interacción entre los factores principales en relación con las variables de motivación. 

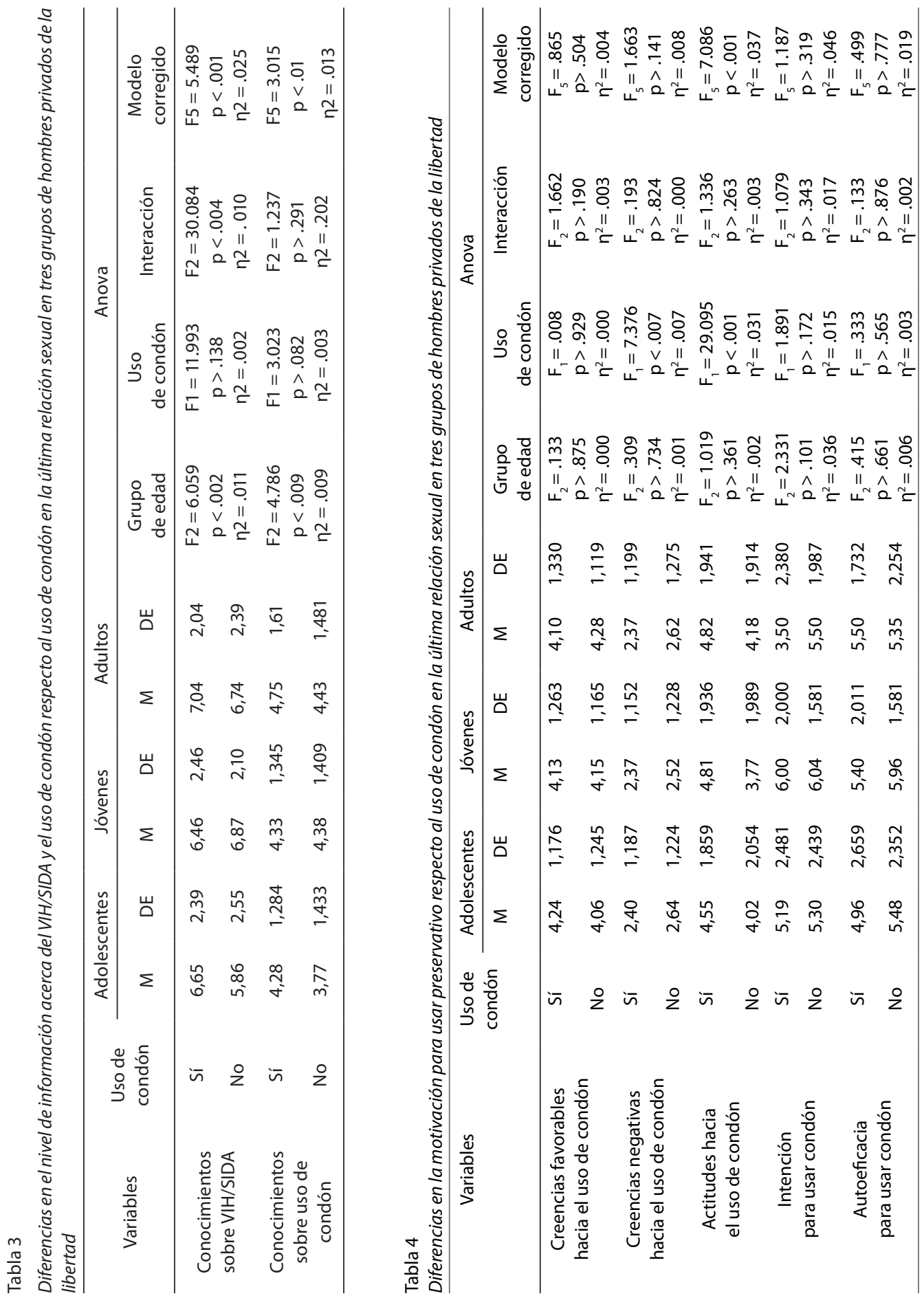
En la tabla 5 se muestran los puntajes obtenidos sobre las habilidades conductuales de los que usaron preservativo en su última relación sexual y los grupos de edad, así como las diferencias al aplicar Anova factorial y eta cuadrada. En la comunicación sobre temas sexuales con la pareja, se observaron diferencias significativas en los grupos de edad y el uso del condón, pero estos dos factores no interactuaron entre sí para explicar la comunicación con la pareja. Al aplicar la prueba Bonferroni, se hallaron diferencias entre adolescentes $y$ jóvenes $(\mathrm{I}-\mathrm{J}=.45, \mathrm{p}<.001)$, y adolescentes y adultos $(\mathrm{I}-\mathrm{J}=.48, \mathrm{p}<.001)$. Estos datos indican que entre más edad tienen, más hablan con la pareja. Los resultados obtenidos sobre los estilos de negociación indican que en el de colaboración-equidad, no hay diferencias en cuanto a la edad, pero si sobre el uso del condón. De igual forma, no se halló un efecto de interacción entre la edad y el uso del condón sobre el estilo de colaboración-equidad. Esto indica que quienes usaron el preservativo utilizan más el estilo de colaboración-equidad, pero la edad no tiene un efecto. Por último, sobre el estilo de contender, se detectaron diferencias significativas entre los grupos de edad, pero no sobre el uso del condón; al igual que en el estilo anterior, no se encontró un efecto de interacción. Al aplicar una prueba post hoc, se observaron diferencias entre adolescentes y adultos $(\mathrm{I}-\mathrm{J}=.38, \mathrm{p}<.001)$, es decir, los más jóvenes imponen su punto de vista en el terreno sexual.

\section{DISCUSIÓN Y CONCLUSIONES}

El objetivo de la presente investigación fue analizar si la información, la motivación y las habilidades conductuales se asocian con el uso o no uso del preservativo en la última relación sexual. Para ello, se comparó a hombres agrupados en tres grupos de edad.

Respecto del nivel de conocimientos, se efectuó la comparación en términos de la información sobre VIH/SIDA y el uso del condón. En líneas generales, los hombres en los tres grupos de edad tuvieron una calificación que osciló entre 5 y 7 sobre lo que sabían de síntomas, transmisión y prevención de VIH/SIDA, y más alto era el nivel de información en los que sí usaron condón. De igual forma, conforme aumentaba la edad, mayor era el nivel de conocimientos. Estos resultados en general coinciden con diversas investigaciones cuando se trata de adolescentes o jóvenes (Cardona, Hernández, Suárez y Zapata, 2015; Del Toro, Gutiérrez, Hoyos, Romero y Sala, 2017; Pérez, Soler, Pérez, López y Leyva, 2016); aunque en población universitaria, el porcentaje de conocimientos fue más alto (Conde et al., 2017; López, Escobar, Hernández y Pérez, 2012). Tales datos dan un panorama de que el nivel de conocimientos es muy similar entre los diferentes grupos de edad, si bien se incrementa cuando son mayores. La información sigue siendo escasa, aunque se ha reportado que no necesariamente el grado de conocimientos se asocia con medidas preventivas (Huapaya y Richard, 2016). Por ello, la información no es garantía, pero sí es necesaria para que las personas sepan por qué deberían protegerse en sus relaciones sexuales (Baiz, Morales y Pereyra, 2016).

Acerca de sus conocimientos sobre el uso del condón en cuanto a qué deben hacer para evitar que se rompa o se deslice en el acto sexual, se observó que sabían 


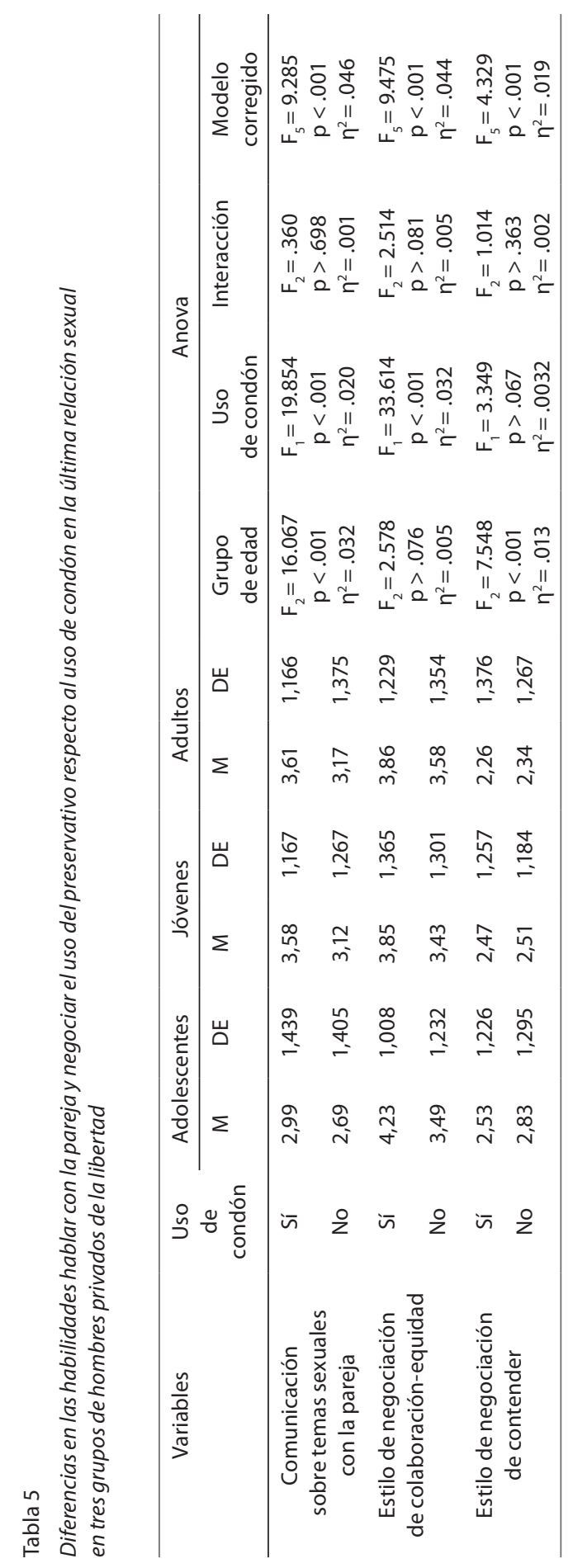


muy poco sobre cómo usarlo; de hecho, las puntaciones oscilaron entre 3 y 4 puntos, en una escala de 0 a 10 . De esta forma, aunque ligeramente la puntuación fue más alta en quienes usaron condón, las diferencias no fueron significativas. Esto es relevante debido a que, aunque algunos de ellos lo utilizan en sus relaciones sexuales, no saber cómo usarlo puede causar que la efectividad del preservativo sea menor y por ende ocasionar algún problema de salud sexual (Cortés, García y Ochoa, 2015).

Con base en lo anterior, es necesario informar a la población sobre cómo prevenir el VIH/SIDA y usar el condón en sus relaciones sexuales. Es el primer paso para lograr que identifiquen el riesgo de contraer el VIH y puedan adoptar una postura de prevención (Amirkhanian, Kelly, Kabakchieva, McAuliffe y Vassileva, 2003).

En el componente del modelo IMB, referente a la motivación, se evaluaron las creencias, actitudes, intención y autoeficacia para usar condón. Sobre estas variables, se encontró que, independientemente del grupo de edad y del uso del preservativo, todos los hombres reportaron tener creencias y actitudes favorables hacia el uso del condón. Consideraban la intención de usar condón en su próxima relación sexual y se sentían capaces de poder usarlo. Estos resultados son congruentes con la literatura, en donde se observa que en términos generales las personas se perciben de esa manera, aunque eso no necesariamente implica que se asocie con el uso del condón (González et al., 2018; Moreno et al., 2008).

Algunos autores mencionan la necesidad de que las personas posean información y estén motivadas para poder llevar a cabo cambios en su comportamiento (Operario, Smith, Arnold y Kegeles, 2010), de tal forma que es probable que por sí solas no den una explicación del uso del condón en la última relación sexual. Además, partiendo del modelo IMB, es necesario el desarrollo de habilidades conductuales (Fisher y Fisher, 1992).

En ese sentido, en la presente investigación se evaluaron algunas habilidades centradas en la comunicación con la pareja sobre temas sexuales y la negociación en el uso del condón, las que han sido documentadas en relación con conductas preventivas, como el uso del preservativo (Ehon, 2008; Jiménez, 2010; Robles, Frías, Moreno, Rodríguez y Barroso, 2011; Sánchez y Enríquez, 2017; Santos Iglesias y Sierra, 2010). De esta forma, se analizó si había diferencias en los hombres en términos de los grupos de edad y sobre el uso de condón. Grosso modo se encontraron diferencias significativas en la frecuencia con que hablaban con su pareja de temas sexuales, incrementándose conforme a la edad y siendo mayor la frecuencia en los que usaron condón.

Como ya se mencionó, estos resultados son congruentes con diversos estudios. Asimismo, se torna relevante que la edad desempeñe un papel en la frecuencia con que se comunican con su pareja. Probablemente, sientan mayor confianza o las experiencias previas han coadyuvado para que hablen más con su pareja y, lo más importante, para usar condón en las relaciones sexuales.

Sobre los estilos de negociación, en el de colaboración-equidad, que refiere a tratar de 
llegar a un acuerdo con la pareja para usar condón, se halló que la edad no era significativa entre los grupos, pero sí con respecto al uso de preservativo, debido a que este estilo fue más utilizado por quienes lo empleaban. En cambio, en el estilo de contender, que indica que las personas imponen su punto de vista y tratan de salirse con la suya para no usar condón, se encontró que no hubo diferencias con respecto a la edad, pero sí sobre el uso del condón, debido a que fue un estilo más utilizado por quienes no lo usaron. Si bien la edad no resulta significativa en relación con los estilos de negociación, se observa que un estilo positivo para usar condón conlleva una conducta preventiva, lo cual puede ayudar a reducir el riesgo de infección por VIH.

En algunos estudios se ha encontrado una estrecha relación entre la negociación para el uso del condón en las relaciones sexuales y la importancia de desarrollar habilidades para que se haga de una manera efectiva (Sánchez, Enríquez y Robles, 2015; Camilleri, Kohut y Fisher, 2015), por lo que es importante considerar que, independientemente de la edad, los hombres reportaron el uso de un estilo de negociación de colaboración-equidad que, si bien se asocia con el empleo del condón, sugiere hacer una evaluación diferente que permita analizar si efectivamente lo utilizan o solo están respondiendo por deseabilidad social (Sánchez, Enríquez, Rosales, Estrada y Lozano, 2018).

Con base en los resultados obtenidos, al comparar a los hombres en términos de edad, considerando la experiencia de vida que han adquirido a lo largo del tiempo, se puede observar que han desarrollado conocimiento y habilidades que pueden impactar en el uso del condón en las relaciones sexuales; sin embargo, al menos en la muestra evaluada, no son suficientes, debido a que la información que poseen es reducida y varios de ellos utilizan un estilo de negociación de uso del preservativo inadecuado. Por ello, es necesario que se entrenen en habilidades para que puedan hablar con su pareja y negociar de manera efectiva el uso del condón.

\section{REFERENCIAS}

Adoga, M., Banwat, E., Forbi, J., Nimzing, L., Pam, C., Gyar, S., ... Agwale, S. (2009). Human Inmunonodeficiency Virus, Hepatitis B Virus and Hepatitis C: Sero-Prevalence, Co-Infection and Risk Factors Among Prison Inmates in Nasarawa State, Nigeria. J Infect Dev Ctries, 3(7), 539-547.

Amirkhanian, Y., Kelly, J., Kabakchieva, E., McAuliffe, T., y Vassileva, S. (2003). Evaluation of a Social Network HIV Prevention Intervention Program for Young Men who Have Sex with Men in Russia and Bulgaria. AIDS Education and Prevention, 15(3), 205-215.

Amuchástegui, A. (2017). Gobernanza neoliberal en la epidemia del VIH/SIDA en mujeres en México: los efectos del paradigma de la vulnerabilidad. Estudios Sociológicos, 35(104), 343-371. 
Baiz, J., Morales, F., y Pereyra, L. (2016). Aspectos psicosociales de la salud sexual y reproductiva en estudiantes universitarios. Salud \& Sociedad, 7(2), 180-195.

Bermúdez, M., Herencia-Leva, A., y Uribe, A. (2009). Versión fuerte y débil del modelo de información-motivación-habilidades conductuales en la predicción del uso de preservativo. Revista Latinoamericana de Psicología, 41(3), 587- 598.

Bórquez, C., Lobato, I., Gazmuri, P., Hurtado, R., Llanqui, V., Vivanco M., ... Vega, J. (2017). Prevalencia de virus de inmunodeficiencia humana, virus de hepatitis B y Treponema pallidum en reclusos del Centro de Detención Preventiva de Arica, Chile. Rer Chilena Infectol, 34(5), 453-457. doi:10.4067/S0716-10182017000500453

Camilleri, M., Kohut, T., y Fisher, W. (2015). Condom Use Behavioural Skills Mediate the Relationship Between Condom Use Motivation and Condom Use Behavior Among Young Adult Heterosexual Males: An Information-Motivation-Behavioural Skills Analysis. The Canadian Journal Sexuality, 24(2), 131-140.

Canche, J., Canul, J., Suárez, R., De-Anda, R., y González, M. (2011). Infecciones por el virus del papiloma humano en mujeres recluidas en centros de readaptación social en el sureste de México. Rev. Esp. Penit, 13, 84-90.

Cardona, J. A., Hernández, J. C., Suárez, G. R., y Zapata, W. (2015). Conocimientos actitudes y prácticas sobre VIH/SIDA en estudiantes de educación media de Medellín. Archivos de Medicina, 11(4), 1-10. doi:10.3823/1272

Conde, M., Hernández, A., Reyes, N, Rosas, D., Taboada, M., Valencia, J., Ruvalcaba, J., y Reynoso, J. (2017). Conocimiento de VIH/SIDA en alumnos de la licenciatura en Farmacia de la Universidad Autónoma del Estado de Hidalgo. Educación y Salud. Boletín Cientifico de Ciencias de la Salud del ICSa, 6(11). Recuperado de https:// repository.uaeh.edu.mx/revistas/index.php/ICSA/article/view/2701/2725

Cortés, A., García, R., y Ochoa, S. (2015). Comportamiento sexual y uso del condón en estudiantes de la Facultad de Tecnología de la Salud. Revista Cubana de Medicina Tropical, 67(2), 202-212.

Cruz, C., Ramos, U., y González, A. (2011). Guía de prevención, diagnóstico y tratamiento de las ITS. Dirigida a personal de servicios de salud. Ciudad de México: Fundación Mexicana para la Salud A.C.

Cueto, S., y Leon, J. (2016). Early Sexual Initiation Among Adolescents: A Longitudinal Analysis for 15-Year-Olds in Peru. Interamerican Journal of Psychology, 50(2), 186-203.

Cunill, M., Gras, M., Planes, M., y Serda, B. (2012). Predictores de la intención de uso del preservativo entre adolescentes. Cuadernos de Medicina Psicosomática y Psiquiatría de Enlace, 104, 9-18.

Del-Toro, A., Gutiérrez, E., Hoyos, A., Romero, A., y Sala, M. (2017). Caracterización del nivel de conocimientos sobre ITS/VIH/SIDA en adolescentes. Multimed. Revista Médica, 19(6), 1006-1019. 
Ehon, A. (2008). Assessing Sexual Communication and Condom Negotiation Among University Undergraduate Students in Sexual Relationship; in the Era of HIV/AIDS. IFE Psychologia, 16(2), 216-239.

Espada, J., Morales, A., Guillén, A., Ballester, R., y Orgilés, M. (2016). Predicting Condom Use in Adolescent: A Test of Three Socio-Cognitive Models Using a Structural Equiation Modeling Approach. BMC Public Health, 16(35), 1-10. doi:10.1186/ s12889-016-2702-0

Fisher, J., y Fisher, W. (1992). Changing AIDS-Risk Behavior. Psychological Bulletin, 3(3), 455-474.

Folch, C., Álvarez, J., Casabona, J., Brotons, M., Castellsagué, X., y Grupo Jóvenes e Internet. (2015). Determinantes de las conductas sexuales de riesgo en jóvenes de Cataluña. Revista Española de Salud Pública, 89, 471-485.

González, J., Morales, D., Murcia, J., Muñoz, N., Ospina, D., y Nossa, L. (2018). Relación entre género y conocimientos, actitudes y prácticas respecto al uso del condón en adolescentes escolarizados, Suba (Bogotá), 2016. Carta Comunitaria, 26(147), 10-16. https://doi.org/10.26752/ccomunitaria.v26.n147.325

Gough, E., y Edwards, P. (2009). HIV Seroprevalence and Associated Risk Factors Among Male Inmates at the Belize Central Prison. Revista Panamericana de Salud Publica, 25(4), 292-299.

Huapaya, G., y Richard, D. (2016). Conocimientos, actitudes y prácticas sobre sexualidad en jóvenes estudiantes de una institución universitaria, Lima, Perú. Revista Muro de la Investigación, 1(1), 97-110.

Jiménez, M. (2010). Comunicación sexual en adolescentes y su implicación en la consistencia del uso del condón. Enseñanza e Investigación en Psicología, 15(1), 107-129.

Kerlinger, F., y Lee, H. (2002). Investigación del comportamiento. Métodos de investigación en ciencias sociales (4. ${ }^{\mathrm{a}}$ ed.). Ciudad de México: McGraw-Hill.

Liu, Z., Wei, P., Huang, M., Liu, Y., Li, L., Gong, X. ... Li., X. (2014). Determinants of Consistent Condom Use Among College Students in China: Application of the Information-Motivation-Behavior Skills (IMB) Model. PLoS ONE, 9(9), 1-9. doi:0.1371/journal.pone.0108976

López, E., Garrido, V., López, J., López, M., y Galvis, M. (2016). Predicción de la reincidencia con delincuentes juveniles: un estudio longitudinal. Revista Española de Investigación Criminológica, 14, 1-22.

López, M., Escobar, F., Hernández, J., y Pérez, C. (2012). Nivel de conocimiento sobre medidas de prevención de VIH-SIDA y las ITS en Estudiantes de la ECR. Revista Colombiana de Rehabilitación, 11(1), 88-95. https://doi.org/10.30788/RevColReh.v11.n1.2012.53

Mazo, Y., Domínguez, L., y Cardona, J. (2014). Conocimientos, actitudes y prácticas en adolescentes universitarios entre 15 y 20 años sobre VIH/SIDA en Medellín, Colombia 2013. Revista Médicas UIS, 27(3), 35-45. 
Moreno, D., Rivera, B., Robles, S., Barroso, R., Frias, B., y Rodriguez, M. (2008). Características del debut sexual de los adolescentes y determinantes del uso consistente del condón desde el análisis contingencial. Psicología y Salud, 18(2), 207-225.

Operario, D., Smith, C. D., Arnold, E., y Kegeles, S. (2010). The Bruthas Project: Evaluation of a Community-Based HIV Prevention Intervention for African American Men who Have Sex with Men and Women. AIDS Education \& Prevention, 22(1), 37-48.

Organización Panamericana de la Salud. (2018). Salud del adolescente-Salud sexual y reproductiva. Recuperado de https://bit.ly/2wLX19

Pérez, R., Soler, S., Pérez, R., López, A., y Leyva, R. (2016). Conocimientos sobre VIH/ SIDA, percepción de riesgo y comportamiento sexual en estudiantes universitarios. Multimed. Revista Médica, 20(1), 1-14.

Programa Conjunto de las Naciones Unidas sobre el VIH/SIDA. (2018). Estadísticas mundiales sobre el VIH de 2017. Recuperado de http://www.unaids.org/sites/default/ files/media_asset/UNAIDS_FactSheet_es.pdf

Robles, S., y Díaz-Loving, R. (2011). Validación de la encuesta estudiantil sobre salud sexual (EESS). Ciudad de México: Facultad de Estudios Superiores Iztacala.

Robles, S., Frías, B., Moreno, D., Rodríguez, M., y Barroso, R. (2011). Conocimientos sobre VIH/SIDA, comunicación sexual y negociación del uso del condón en adolescentes sexualmente activos. Revista Electrónica de Psicología Iztacala, 14(4), 317-340.

Sánchez, R., y Enríquez, D. (2017). Factores asociados a la consistencia en el uso del condón en adolescentes en conflicto con la ley. Psicología Iberoamericana, 25(1), 78-86.

Sánchez R., Enríquez D., y Robles S. (2015). Evaluación de un programa de intervención para promover la salud sexual en adolescentes mexicanos en conflicto con la ley. Acta Colombiana de Psicología, 19(2), 257-268. doi:10.14718/ACP.2016.19.2.11

Sánchez, R., Enríquez, D., Rosales, R., Estrada, M., y Lozano, M. (2018). Negociación y uso correcto del condón en hombres privados de la libertad. En R. Díaz-Loving, I. Reyes-Lagunes y F. López. Aportaciones actuales de la psicología social (vol. IV, pp. 2659-2674). Ciudad de México: Amepso.

Sánchez, R., Rosales, R., y Enríquez, D. (2018). Factores familiares asociados a conductas de riesgo en hombres con y sin conflicto con la ley. En R. Díaz-Loving, I. Reyes-Lagunes y F. López. La psicología social en México (vol. XVII, pp. 911-927). Ciudad de México: Amepso.

Santos-Iglesias, P., y Sierra, J. C. (2010). El papel de la asertividad sexual en la sexualidad humana: una revisión sistemática. International Journal of Clinical and Health Psychology, 10(3), 553-577.

Uribe, J., Aguilar, J., Zacarías, X., y Aguilar, A. (2015). Modelos explicativos del uso del condón en las relaciones sexuales de adolescentes. Acta de Investigación Psicológica 5(1), 1904-1925. 